\begin{abstract}
Allan Alves Valeriano
Um mecanismo de seleção de componentes para o middleware Kaluana usando a noção de contratos

de reconfiguração

Dissertação de Mestrado

Dissertação apresentada como requisito parcial para obtenção do título de Mestre pelo Programa de PósGraduação em Informática da PUC-Rio.
\end{abstract}

Orientador: Prof. Markus Endler 
Allan Alves Valeriano

\title{
Um mecanismo de seleção de componentes para o middleware Kaluana usando a noção de contratos de reconfiguração
}

\begin{abstract}
Dissertação apresentada como requisito parcial para a obtenção do grau de Mestre pelo Programa de Pósgraduação em Informática do Departamento de Informática do Centro Técnico e Científico da PUCRio. Aprovada pela Comissão Examinadora abaixo assinada.
\end{abstract}

Prof. Markus Endler

Orientador

Departamento de Informática - PUC-Rio

Prof.- Noemi de La Rocque Rodriguez

Departamento de Informática - PUC-Rio

Prof. Renato Fontoura de Gusmão Cerqueira

Departamento de Informática - PUC-Rio

Prof. José Eugenio Leal

Coordenador Setorial do Centro Técnico Científico - PUC-Rio 
Todos os direitos reservados. É proibida a reprodução total ou parcial do trabalho sem autorização da universidade, do autor e do orientador.

\section{Allan Alves Valeriano}

Engenheiro de Computação formado pela Pontifícia Universidade Católica do Rio de Janeiro (PUC-Rio) em 2007. Atualmente, integra o grupo de pesquisadores do LAC (Laboratory for Advanced Collaboration) da PUCRio, desenvolvendo pesquisa na área de Sistemas Distribuídos.

Ficha Catalográfica

Valeriano, Allan Alves

Um mecanismo de seleção de componentes para o middleware Kaluana usando a noção de contratos de reconfiguração/ Allan Alves Valeriano; orientador: Markus Endler.

87 f. : il. ; $30 \mathrm{~cm}$

Dissertação (Mestrado em Informática)-Pontifícia Universidade Católica do Rio de Janeiro, Rio de Janeiro, 2009.

Inclui bibliografia

1. Informática - Teses. 2. Adaptação dinâmica. 3. Contrato de reconfiguração. 4. Componentes de software. 5. Middleware. I. Endler, Markus. II. Pontifícia Universidade Católica do Rio de Janeiro. Departamento de Informática. IV. Título. 


\section{Agradecimentos}

À Juliana Aquino, por toda sua paciência e apoio durante o desenvolvimento deste trabalho, sem os quais não seria possível sua conclusão.

Ao Professor Markus Endler, por sua compreensão e orientação durante todo o mestrado.

Ao Nima por fornecer o mapa detalhado do campus da PUC-Rio para ser usado na aplicação exemplo deste trabalho.

Ao CNPq, à PUC-Rio, ao Departamento de Informática e ao Tecgraf, pelos auxílios financeiros concedidos, sem os quais este trabalho não poderia ter sido realizado. 


\section{Resumo}

Valeriano, Allan Alves; Endler, Markus. Um mecanismo de seleção de componentes para o middleware Kaluana usando a noção de contratos de reconfiguração. Rio de Janeiro, 2011. 87 p. Dissertação de Mestrado Departamento de Informática, Pontifícia Universidade Católica do Rio de Janeiro.

A computação móvel cria a necessidade de que as aplicações sejam adaptáveis de acordo com o contexto do usuário. Demandas específicas do usuário, assim como alterações no contexto computacional requerem que aplicações móveis clientes devam ser capazes de se adaptar dinamicamente para se adequar ao novo cenário de execução. Estas adaptações precisam ser apropriadas e devem manter a qualidade de serviço evitando falhas ou degradação do desempenho da aplicação. Este trabalho propõe uma extensão do middleware de componentes Kaluana, que provê um mecanismo de seleção de componentes para aplicações adaptáveis a partir de contratos de reconfiguração. Esta seleção é feita com a noção de equivalência entre suas as interfaces públicas e considera as restrições de execução dos componentes candidatos a serem usados na adaptação de acordo com o contexto computacional do dispositivo para a avaliação dos componentes candidatos a instanciação. Esta seleção visa manter a compatibilidade dos novos componentes com os componentes já implantados bem como com o contexto de execução, ou seja, o estado corrente dos recursos no dispositivo. Com a noção de equivalência entre as interfaces de especificação de componentes, a aplicação tem a possibilidade de selecionar componentes através da interface do serviço requisitado sem a necessidade de conhecer seu nome ou alguma característica específica, evitando assim que uma amarração seja criada com uma determinada implementação.

\section{Palavras-chave}

Adaptação dinâmica; Kaluana; Componentes de software; Middleware. 


\section{Abstract}

Valeriano, Allan Alves, Endler, Markus (Advisor). A mechanism of component selection with the notion of reconfiguration contracts. Rio de Janeiro, 2011. 87 p. MSc. Dissertation - Departamento de Informática, Pontifícia Universidade Católica do Rio de Janeiro.

Mobile computing creates the need for applications to be adaptable according to the user's context. Specific user demands as well as changes in the computational context the mobile applications require clients to be able to adapt dynamically to suit the new scenario of execution. These adjustments should be appropriate and should maintain the quality of service avoiding failures or preventing degradation of application performance. This thesis proposes an extension of Kaluana middleware which provides a mechanism for selection of components for adaptive applications based on the notion of reconfigurations contracts. This selection is done also based on the notion of equivalence between their public interfaces and should consider the execution restrictions of the candidate components to be used in the adaptation in accord to the device's execution context to be used to evaluate the candidate components to the instantiation. This selection aims to maintain the compatibility of new components with the components already used as well as the execution context, i.e. the current status of the device's resources. Due to the notion of equivalence between the interface specifications of components, the application should be able to request a component through the interface of the requested service, avoiding the need to know the component's name or any other specific feature that creates a tie with the given implementation.

\section{Keywords}

Dynamic adaptation; Kaluana; Software components; middleware. 


\section{Sumário}

1 Introdução 12

1.1. Motivação e contribuição 12

1.2. Estrutura da dissertação 14

2 Fundamentos $\quad 15$

2.1. Componentes 15

2.2. Ciência ao contexto 16

2.3. Descrição de interfaces 17

2.4. Compatibilidade de tipos 19

2.5. Descrição de requisitos 20

2.6. Políticas de adaptação 21

2.7. Adaptação dinâmica 22

2.8. Arquiteturas orientadas a serviço 25

2.9. Gerenciamento de serviços 27

2.10. Mobilis 28

2.10.1. Shared Data Manager 29

2.10.2. Context Management Service 30

3 Kaluana 31

3.1. Arquitetura 31

3.2. Ciclo de vida 33

4 Trabalhos Relacionados $\quad 36$

4.1. CASA 36

4.2. IAM 38

4.3. Chisel 43

4.4. DynamicTAO 45 
5 Mecanismo de seleção de componentes $\quad 50$

5.1. Restrições computacionais para a execução de um componente 51

5.2. Contrato de reconfiguração de um componente 54

5.3. Integração do Kaluana com a plataforma Mobilis 55

5.4. Registro de componentes no Kaluana $\quad 57$

5.5. Requisição de um componente por uma aplicação 58

5.6. Equivalência de componentes 60

5.7. Download e instalação de componentes, a partir de um servidor remoto

61

6 Resultados experimentais $\quad 64$

6.1. Testes de desempenho 64

6.1.1. Busca de componentes $\quad 65$

6.1.2. Requisições múltiplas $\quad 66$

6.2. Aplicação exemplo $\quad 67$

6.3. Casos de uso 69

6.3.1. Ator percorre uma área não demarcada 69

6.3.2. Ator entra em uma área demarcada 70

6.4. Middleware Kamaiurá 73

6.5. Guideline de criação de uma aplicação dinâmica usando o Kaluana 76

$\begin{array}{ll}7 \text { Conclusões } & 79\end{array}$

7.1. Limitações 80

7.2. Trabalhos futuros $\quad 80$

7.2.1. Uso de ontologias para descrição e seleção de componentes 81

7.2.2. Flexibilização do uso de interesses ao contexto 81

7.2.3. Flexibilização da descoberta de servidores remotos 82

8 Referências Bibliográficas 83 


\section{Lista de figuras}

Figura 1. Representação da composição de dois componentes 16

Figura 2: Classificação dos tipos de adaptação dinâmica, segundo [12]..24

Figura 3. Arquitetura em camadas, da plataforma Mobilis ........................... 29

Figura 4. Arquitetura do middleware Kaluana, segundo [12] ........................32

Figura 5. Métodos de reconfiguração de componentes [10] .........................37

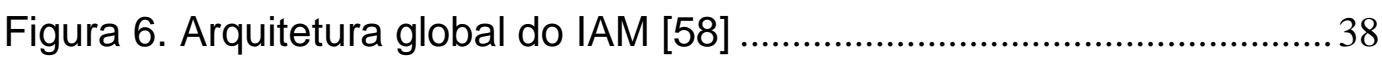

Figura 7. Exemplo de um metaobjeto no IAM,

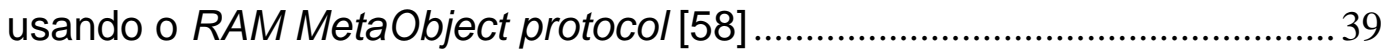

Figura 8. Camada que trata de requisitos não funcionais, no IAM [58] ..... 40

Figura 9. Organização do framework de

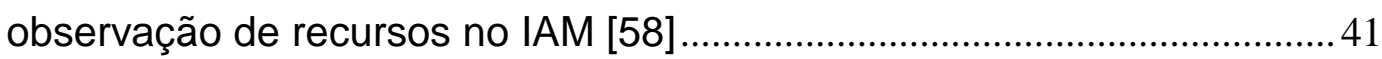

Figura 10. Políticas de adaptação no IAM [58] ................................................ 42

Figura 11. Linguagem definição de políticas

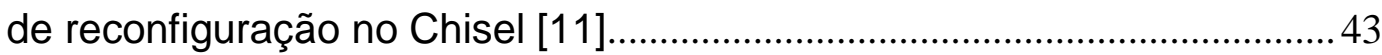

Figura 12. Mecanismo de adaptação do Chisel [11].....................................45

Figura 13 Mecanismo de especialização do DynamicTAO [65]................... 46

Figura 14 Arquitetura do DynamicTAO [65] ..................................................4

Figura 15. Definição do contrato de reconfiguração de um componente.. 55

Figura 16. Registro do consumidor de

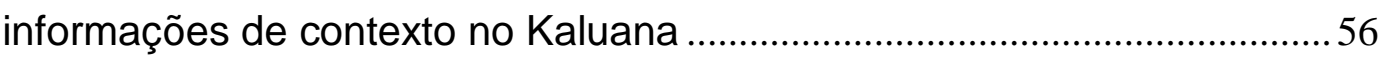

Figura 17. Exemplo de registro de um componente no Kaluana.................57

Figura 18. Declaração de um componente no manifesto do Android.........59

Figura 19. Requisição de um componente por nome ....................................59

Figura 20. Requisição de um componente

pelo nome de um serviço necessário .............................................................59

Figura 21 Instalação de um componente novo no dispositivo .......................62

Figura 22 Usuário escolhe se deseja usar a aplicação instalada ou não.. 62

Figura 23 Requisição de componentes sequencialmente .............................66 
Figura 24 Requisição paralela de componentes .........................................67

Figura 25. Componente default, GoogleMapComponent ........................... 68

Figura 26. Requisição de carregamento de um novo componente............ 69

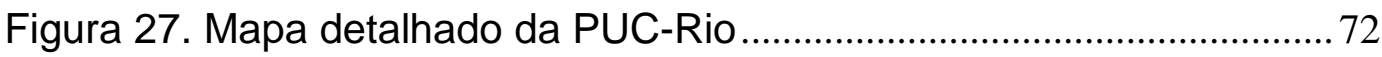

Figura 28. Arquitetura do Kamaiurá sobre o middleware Kaluana............. 74

Figura 29 Realizando o bind de uma aplicação

no serviço de gerenciamento de componentes do Kaluana........................77

Figura 30. Registro do receiver de notificação

do carregamento dos componentes requisitados. 78 